\title{
Arterial Thoracic Outlet Syndrome - A Rare Entity
}

Rajul Rastogi ${ }^{1 *}$, Shourya Sharma ${ }^{1}$, Ankit Singhal ${ }^{1,2}$, Prabhat Kumar Bhagat ${ }^{1}$, Yuktika Gupta ${ }^{1}$, Pankaj Kumar Das1, Mohini Choudhary ${ }^{1}$ and Vijai Pratap ${ }^{1}$

${ }^{1}$ Department of Radiodiagnosis, Teerthanker Mahaveer Medical College and Research Center, Moradabad, UP, India

${ }^{2}$ Consultant Neurophysician, Moradabad, UP, India

*Corresponding author: Rajul Rastogi, Department of Radiodiagnosis, Teerthanker Mahaveer Medical College and Research Center, Moradabad, U.P India, E-mail: eesharastogi@gmail.com

Received date: Nov 24, 2016; Accepted date: Nov 28, 2016; Published date: Nov 30, 2016

Copyright: (c) 2016 Rastogi, et al. This is an open-access article distributed under the terms of the Creative Commons Attribution License, which permits unrestricted use, distribution, and reproduction in any medium, provided the original author and source are credited.

\begin{abstract}
Thoracic outlet syndrome refers to a constellation of neurovascular signs and symptoms caused by compression of neural and vascular structures as they exit through the superior thoracic aperture to enter the upper limb. Arterial type is the rarest and requires early diagnosis \& management to prevent severe complications like thromboembolic phenomenon. Hence, in this article, we are describing thoracic outlet syndrome arising due to compression of right subclavian artery that was diagnosed by imaging.
\end{abstract}

Keywords: Arterial; Thoracic; Outlet; Color-Doppler

\section{Case report}

A 35-year old male with 2-3 months' history of numbness and paresthesia in right upper limb following an overhead smash-shot during badminton play came to our department for Computed Tomography Angiography (CTA). Though clinical examination revealed feeble radial pulse ipsilaterally yet there was no discoloration of skin and other signs of ischemia. All routine laboratory tests were unremarkable.

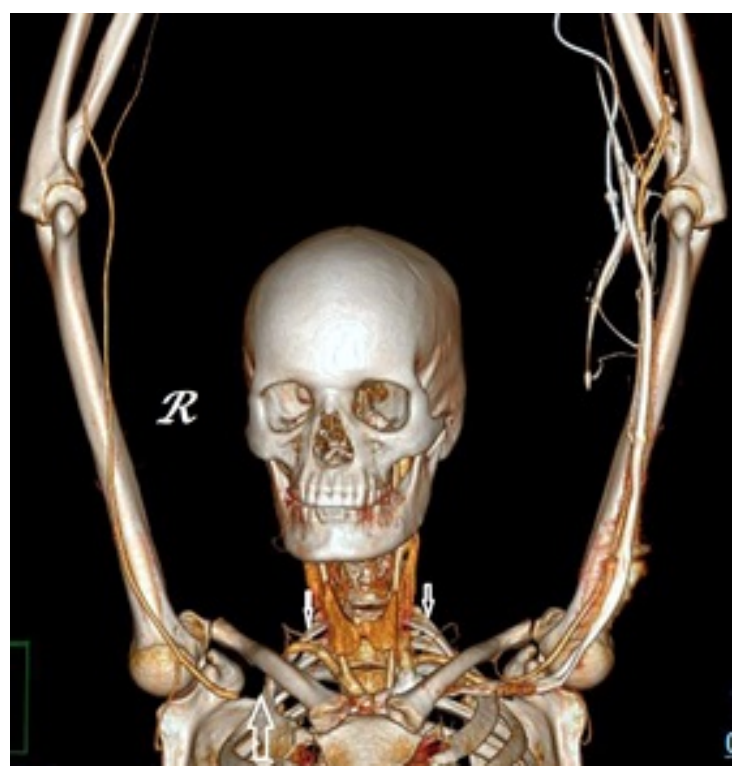

Figure 1: CTA VRT image showing nonvisualised portion of distal right SCA (large arrow) with normal appearing right axillary \& brachial arteries and bilateral cervical ribs arising from $\mathrm{C} 7$ vertebrae (thin small arrows).

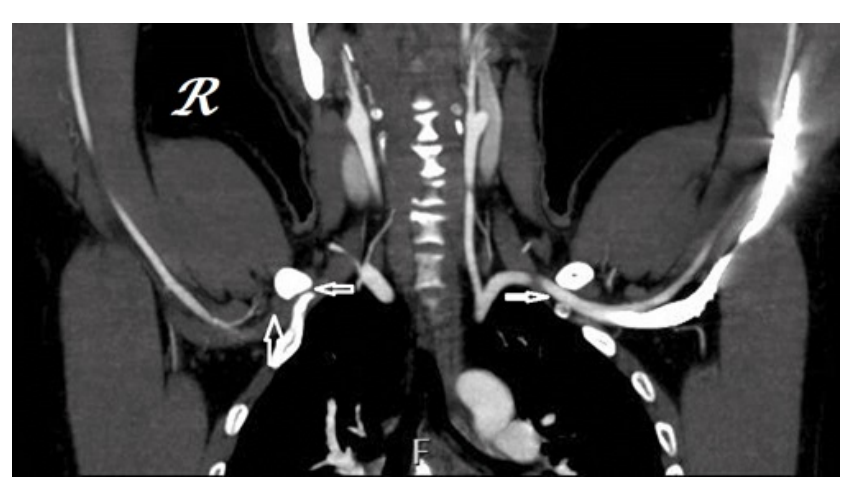

Figure 2: CTA MIP image in coronal plane shows nonvisualised portion of distal right SCA (large arrow) with narrowing of costoclavicular space on right side relative to left side (thin small arrows).

CTA revealed nonvisualisation of short-segment of distal part of right subclavian artery in the region of thoracic outlet associated with narrowing of ipsilateral costoclavicular space caused by synostosis of cervical rib arising from C7 vertebra with first dorsal rib (Figures 1 and 2 ). No evidence of any obvious post-stenotic dilation or collateral channels or space occupying lesion was noted. Axillary and rest of the arteries of right upper limb were normally visualised but were slightly less in caliber relative to contralateral side. 


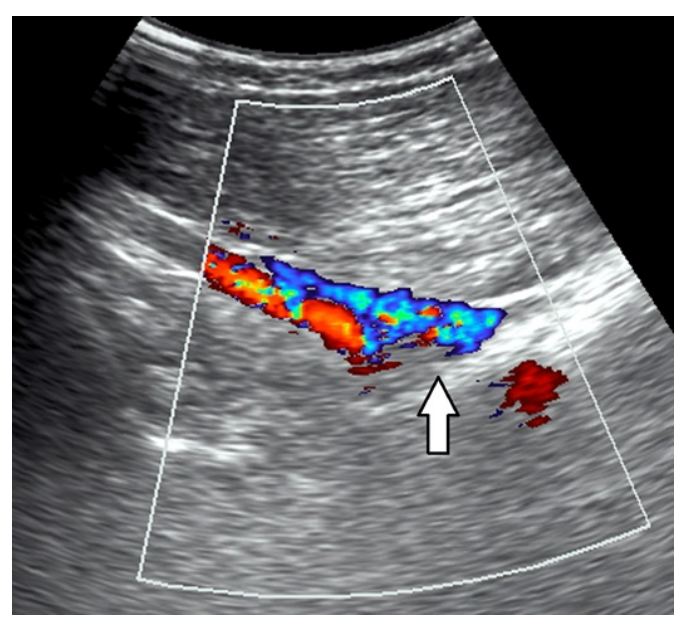

Figure 3: Color Doppler US image with overhead abduction of arm shows nonvisualised distal right SCA (arrow).

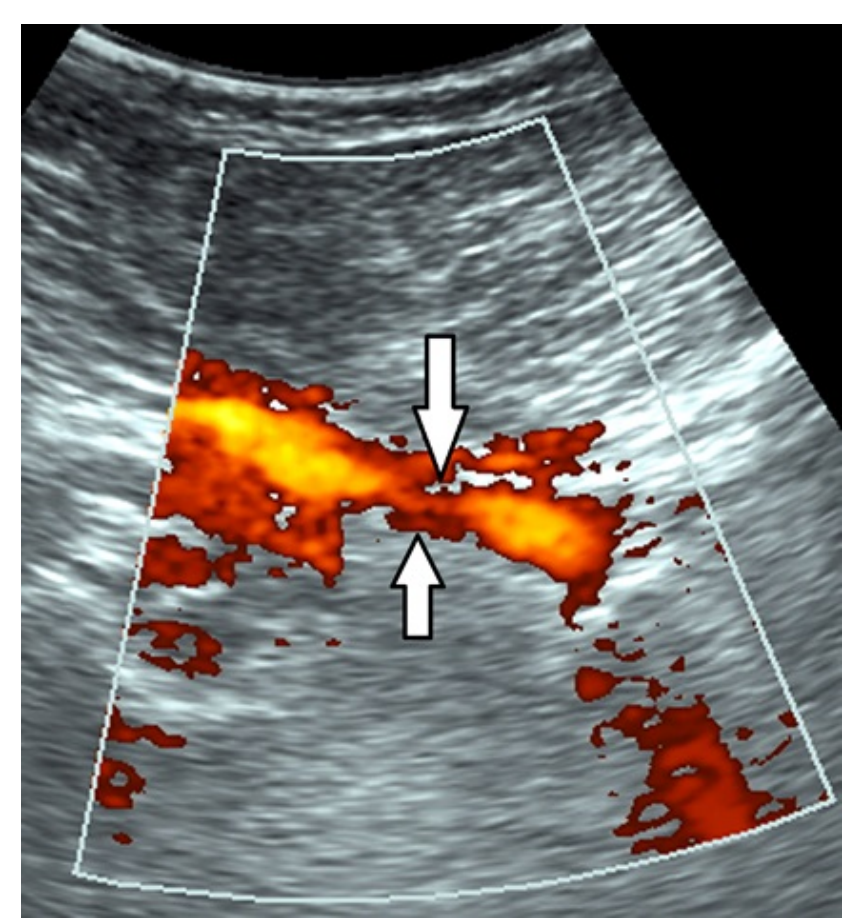

Figure 4: Power Doppler US image in adducted arm position shows narrow segment of distal right SCA corresponding to the nonvisualised segment in figure 3 (in between the arrows).

As patient head no clinical signs of ischemic changes, he was then taken up for Color Doppler Imaging (CDI) for getting more information about the nonvisualised segment of right subclavian artery. CDI revealed luminal narrowing of the corresponding nonvisualised segment of right subclavian artery; being more marked in overhead position of the right arm (Figures 3 and 4). This made us realise that the nonvisualisation of subclavian artery on CTA was secondary to the overhead position of right arm.
Based on the above radiological findings, the diagnosis of arterial thoracic outlet syndrome (ATOS) secondary to costoclavicular space stenosis was made.

\section{Discussion}

Thoracic outlet syndrome (TOS) is a clinical entity characterised by signs \& symptoms arising secondary to compression of brachial plexus or subclavian vein or subclavian artery (SCA) as they exit from superior thoracic aperture to enter the upper limb. It may be congenital or acquired and may present with neural or vascular symptoms or combination of both [1]. Thoracic outlet extends from the cervical spine \& mediastinum to the lower border of pectoralis minor muscle. The syndrome is four times commoner in females than males and is seen between 3rd to 5th decades [1].

The three common locations of compression of neurovascular bundle of the upper limb at the thoracic outlet include scalene triangle (space between scalenus anterior \& medius muscles); costoclavicular space (space between coracoid process \& clavicle) and retro or subpectoral space (space between pectoralis minor muscle \& coracoid process).

Commonest etiology of TOS is scalenus anticus syndrome characterised by abnormal insertion of scalenus anterior muscle on 1st rib followed by cervical rib; abnormalities of 1 st rib or clavicle (1st rib uniting with another rib instead of sternum, malunited fracture, abnormal callus following fracture, Paget's disease, tumors like exostosis); abnormally large C7 vertebral transverse process (tip of C7 transverse process extending beyond the limits of D1 transverse process); muscular hypertrophy; supraclavicular adenopathy, etc. [1-3].

Neurogenic type of TOS is commonest (more than 90\%) while arterial type is rarest (1-3\%) with former presenting with pain, paresthesia \& numbness of upper limb [2]. Venous type is commonly associated with thrombosis of subclavian vein presenting with pain \& swelling of upper limb while arterial type is characterised by signs of ischemia like coolness, pallor, claudication, paresthesia and reduced upper limb pulses. Our case is unique in the fact that he presentation occurred for the first time in 4 th decade by an identifiable precipitating factor similar to one described by Esposito MD et al in a ball thrower [4]. Probably the overhead smash shot during badminton play caused movement at the abnormal union between the cervical \& first dorsal ribs resulting in narrowing of right costoclavicular space below a critical level sufficient to cause symptoms.

Imaging in adducted \& abducted position of arm (latter above the level of head) using CDI, CT, magnetic resonance imaging (MRI) or angiography can be variably useful depending upon the clinical setting [1-5]. CDI is primarily useful in venous \& arterial type of TOS and has the advantage of being dynamic, less time-consuming, noninvasive \& radiation-free. CT scan is primarily useful in vascular type of TOS as it can be combined with angiography and provides information about the etiology especially bony pathology but has the disadvantage of radiation exposure and contrast-related side effects. MRI is useful in both neural \& vascular type of TOS as it can be combined with angiography and provides nearly all the information needed and also has the advantage of being noninvasive, radiation-free and reproducible but has the disadvantage of being expensive \& more timeconsuming [6]. Thus, MRI is the single stop-shop for imaging of TOS.

Imaging in ATOS may reveal transient stenosis on overhead abduction of arm or fixed stenosis of SCA at the site of dynamic 
Citation: Rastogi R, Sharma S, Singhal A, Bhagat PK, Gupta Y, et al. (2016) Arterial Thoracic Outlet Syndrome - A Rare Entity. J Neurol Disord

compression caused by osseous or soft tissue abnormalities with or without associated complications like thrombosis; distal embolism; collaterals \& / or aneurysm / pseudoaneurysm of SCA.

Management of ATOS is primarily surgical excision of cervical \& 1st dorsal rib to prevent as well as treat thromboembolic phenomena. In acute cases, endovascular removal of clot may be needed to prevent complications.

\section{Conclusion}

Imaging plays a key role in diagnosis and management of TOS with CDI \& CTA being complimentary to each other in vascular type while MRI being not only the gold standard imaging technique in neurogenic type but is also a single-stop shop for all cases with suspected TOS. Hence, knowledge of imaging features is a key to early diagnosis.

\section{References}

1. Buller LT, Jose J, Baraga M, Lesniak B (2015) Thoracic Outlet Syndrome: Current Concepts, Imaging Features, and Therapeutic Strategies. Am J Orthop 44: 376-382.
2. Raptis CA, Sridhar S, Thompson RW, Fowler KJ, Bhalla S, et al. (2016) Imaging of the Patient with Thoracic Outlet Syndrome. Radiographics 36: 984-1000.

3. Demondion X, Herbinet P, Van Sint Jan S, Boutry N, Chantelot C, et al. (2006) Imaging assessment of thoracic outlet syndrome. Radiographics 26: 1735-50.

4. Esposito MD, Arrington JA, Blackshear MN, Murtagh FR, Silbiger ML, et al. (1997) Thoracic outlet syndrome in a throwing athlete diagnosed with MRI and MRA. J Magn Reson Imaging 7: 598-599.

5. Remy-Jardin M, Remy J, Masson P, Bonnel F, Debatselier P, et al. (2000) Helical CT angiography of thoracic outlet syndrome: functional anatomy. AJR Am J Roentgenol 174: 1667-1674.

6. Stepansky F, Hecht EM, Rivera R, Hirsh LE, Taouli B, et-al. (2008) Dynamic MR Angiography of upper extremity vascular disease: pictorial review. Radiographics 28: e28. 